\title{
Significado y valores de los sufijos en la toponimia ribagorzana
} aragonesa

Moisés Selfa Sastre

\section{Citer ce document / Cite this document :}

Selfa Sastre Moisés. Significado y valores de los sufijos en la toponimia ribagorzana aragonesa . In: Nouvelle revue d'onomastique, $n^{\circ} 53,2011$. pp. 65-78;

doi : https://doi.org/10.3406/onoma.2011.1725

https://www.persee.fr/doc/onoma_0755-7752_2011_num_53_1_1725

Fichier pdf généré le 04/04/2018 
Significado y valores de los sufijos en la toponimia ribagorzana aragonesa

\author{
Moisés Selfa Sastre \\ (Universitat de Lleida, Cataluña, España)
}

\title{
Significado y valores de los sufijos en la toponimia ribagorzana aragonesa
}

La toponimia aragonesa, románica en su conjunto y de la cual forma parte la ribagorzana, ha atraído la atención de numerosos investigadores desde mediados del siglo $\mathrm{XX}$ con trabajos dedicados al estudio de los nombres de lugar desde una amplia perspectiva sincrónica y diacrónica. Entre estos investigadores tenemos que destacar los nombres de M. Alvar (1949), A. Badía (1949), W. D. Elkock (1949), M. García Blanco (1949) y, más recientemente, los de G. Francino $(2002,2005,2011)$, J. M. DE las Pueblas (2002), C. A Rizos (2001), J. A. Saura (2008), M. Selfa (2003), J. TERRADO $(1992,1998)$ y J. VÁZQUEZ OBRADOR $(2002,2008)$.

El nombre del Dr. J. Terrado, profesor de la Universitat de Lleida, está estrechamente relacionado a un Proyecto de Investigación iniciado por este investigador en el año 1995, desde su propia Universidad, y que lleva el título de Toponimia de Ribagorza ${ }^{l}$. La finalidad de esta investigación es muy clara: recopilar toda la toponimia ribagorzana mediante encuestas orales y analizarla desde el punto de vista de la lingüística sincrónica y diacrónica. A día de hoy, y después de 26 años de investigación, podemos hablar de la publicación de 34 títulos $^{2}$ de los municipios que conforman la Ribagorza aragonesa y catalana y en los que se recoge y analiza toda la toponimia de esta amplia comarca histórica. Desde este punto de vista, y aprovechando los materiales onomásticos publicados ${ }^{3}$, el objetivo de nuestro trabajo será estudiar y establecer los valores y significados de los sufijos en la toponimia de la Ribagorza aragonesa ${ }^{4}$.

\footnotetext{
${ }^{1}$ El Proyecto de Investigación Toponimia de Ribagorza (PS94-0208), al cual pertenecemos como investigador de la Universitat de Lleida, es financiado desde el año 1995 hasta el 1998 por la Dirección General de Investigación Científica y Técnica (Ministerio de Educación y Ciencia). Junto a esta subvención, se buscaron otras fuentes de financiación que han permitido la consecución de los objetivos finales del Proyecto de Investigación. Entre las entidades financiadoras hay que destacar la Diputación General del Gobierno de Aragón, el Instituto de Estudios Altoaragoneses, l'Institut Cartogràfic de Catalunya y el Ayuntamiento de Lérida.

${ }^{2}$ Véase en la bibliografía final los 34 títulos a los que nos referimos.

${ }^{3}$ Téngase en cuenta que muchos de los topónimos que citaremos pertenecen al léxico particular de las hablas ribagorzanas. Para conocer su significado será necesario consultar los vocabularios de ANDOLZ (1992), BALLARÍN (1978), MOTT (1984) y ROHLFS (1985).

${ }^{4}$ La denominación de Ribagorza refiere un amplio espacio geográfico de las provincias de Huesca (Aragón) y también Lérida (Cataluña) en base a las costumbres, la historia y los vínculos socioculturales entre las poblaciones que lo conforman. Tradicionalmente se habla de la Alta Ribagorza, cuya capital es el Pont de Suert, y la Baja Ribagorza, con capital en Graus.
} 


\section{La sufijación.}

La sufijación es un procedimiento de creación léxica abundantísimo en toda nuestra amplia zona de estudio. Como dice la profesora M. L. ARnAL (1986, p. 67-88), la información semántica que aportan los sufijos no es siempre objetivo-nocional o subjetivo-emocional, sino que aportan matices afectivos, expresivos, aumentativos y diminutivos que refuerzan todavía más el significado de la base léxica a la que se unen ${ }^{5}$. Nosotros hemos documentado cuarenta tipos de sufijos diferentes que presentamos alfabéticamente. Para cada sufijo indicamos el valor o los valores semánticos que hemos anotado y, entre paréntesis, el número de la monografía ${ }^{6}$ en la que éste aparece.

1.1. -acho, -acha(s) (<aceu?): respecto al origen de este sufijo se ha planteado cierta controversia, pues se han propuesto diversas procedencias. BUESA (1989) opina que probablemente sea de origen mozárabe; KUHN (1978), por el contrario, indica que quizás venga del latín -aticu, y ALVAR (1953), del mismo modo que ROHLFS (1988), considera que tiene un origen desconocido, a pesar de que apunta la posibilidad de que derive de -асе̌u (GIRALT 1995: 232).

Actualmente es muy poco productivo en la toponimia de nuestra zona de estudio. Los ejemplos recogidos parecen otorgarle un valor aumentativo: Abichachas (3, 23), Corvacho (14), Covacho (23), Covaracho (25).

1.2. -a(d)o, -ada(s) / -ades, -ado, -at, -ata, -áu (<-ātu, -āta): es uno de los sufijos más productivos de la toponimia ribagorzana aragonesa. Como indica MONGE (1965, p. 230) su valor primitivo y fundamental es el de formar abstractos de acción, sentido del cual derivan todos los otros. siguientes:

Los valores semánticos que tiene este sufijo en nuestra toponimia son los

1.2.1. Indica el resultado de la acción del verbo: Afumao (3), Ascllatada (32), Batallats (14), Baixada (22), Cremadas (26), Monte Comprado (6), Molino Espallau (6), Foradada (13), Marrada(s) (3, 12, 18), Mascaradas (25), Pedras Pintadas (6), Puyada (18), Quebrada (27), Fuen Salada (23), Ruñada (6), Socarráu (22), Sucarradas (4, 6, 14, 20), Tallada(s) (3, 5), Tancao (9) / Tancau (12, 25, 27), Trabada (18), Vedao (3, 23) / Vedau $(12,19,23,27)$.

1.2.2. Marca la idea también de colectividad o conjunto del primitivo: Aiguáu (27), Asclláu (27), Bancaladas (28, 31) / Bancalades (31), Baronado (22), Barronada (29), Brocada (7, 13), Burrugat (8), Cabasteradas (27), Clatadas (28), Collada (6, 19 ,

\footnotetext{
${ }^{5}$ El valor semántico de los sufijos en español es una cuestión tratada magistralmente por autores clásicos como A. AlONSo (1967) y también F. MONGE (1965). A pesar de los años transcurridos desde la publicación de estos dos títulos, todavía continúan siendo de especial importancia para el estudio de la sufijación románica.

${ }^{6}$ El listado de las monografías que sobre toponimia ribagorzana se han publicado hasta la fecha puede consultarse en la bibliografía final. Transcribimos literalmente cada topónimo según la ortografía utilizada por el autor de la monografía en cuestión. Eso quiere decir que nos encontraremos con dualidades como, por ejemplo, Pletil / Plletil.
} 
Significado y valores de los sufijos en la toponimia ribagorzana aragonesa

23, 29), Escorcilladas (27), Espigadas (27), Espigolada (12), Esponada (6), Femadas (26), Forcat (5), Manada (3), Mayolada (13) / Mallolada (19, 23, 24), Paretadas (17), Pezolata (27), Pitolada (2), Planada (6, 18), Rebollada (8), Sarrado (7) / Sarrao (18, 23) / Sarrau (3, 6, 23, 14, 25, 27, 28) / Serrao (3, 8), Sarrat (12), Serrat (8, 21), Soladas (18), Terrenadas (9), Trefogats (8), Varada (32), Vivats (5).

1.3. -al(s) (<-āle): como afirma ALVAR (1953, p. 258-259) presenta idénticos valores en castellano, catalán, aragonés y gascón. Son los siguientes:

1.3.1. señala lugares poblados de determinados árboles y plantas: Avenal (27), Barzals (27), Carrascal (1, 12, 15, 23), Carabazals (12), Cerezal (12), Cibadals (3, 15, 28), Cirezal (12), Corniarals (23), Cornierals (23), Chinebral (23, 25), Figueral (15), Freixenal (14), Malloals (23) Nogals (12), Noguerals (14), Ortials (15), Parral(s) (8, 15, 26), Pinarral (8), Romeral (3), Sabinal (3, 13, 23), Vinyal (5).

1.3.2. Suele tener un valor locativo juntamente con el sentido de ampliación y abundancia: Aguals (28), Arenals (13), Bañals (23), Arenal (12, 25), Arnedals (12), Arnerals (12), Bassal (30), Brañals (10), Branyinals (29), Brocal (3), Campanals (5), Cantal (8), Cantals (8), Cequial(s) (23, 24), Cibadal (29), Corñeral (24), Coronal (24), Charcal (14), Chinebral (24), Esponal(s) (15, 26, 28, 31), Farrañal (13) / Ferrañals (7), Fontanal(s) (14, 18, 22, 23, 26), Fosal (24), Ginebral (29), Hortals (23), Montal (7), Morral (4, 21), Nuguerals (19), Pardinals (23, 27), Pedregal(s) (19, 20, 26), Pitral (29), Portals (8), Puyals (8), Riberal (28), Romeral (30), Sarradal(s) (13, 23, 28), Tarteral (14), Terrerals (23), Tormegals (23), Viñals $(6,20)$.

1.3.3. También ha originado sustantivos que indican cualidad, semblanza y pertenencia: Arnal (23), Bancal(s) (6, 23), Casal (21), Comenals (12), Comunal (8, 12, 19, 23, 31), Corral (25), Hortal (20), Portal (26), Puntals (23), Puyal (8, 12, 23), Regal (8), Rabassal (2), Tancal (23), Tozal (3, 9, 23) / Tuzal (3, 9, 12, 18, 23), Vaquerisal (14), Viarals (2).

1.4. -allo, -alls, -allon(s) (<-acŭlu): de escasa productividad, aparece formando, mayoritariamente, nombres de instrumentos y utensilios (GIRALT 1995, p. 228), si bien en algún caso tiene ofrece también un valor despectivo: Bruballa (13), Campallons (14), Cornalla (10), Forcallo (3, 9, 12, 18, 23, 25), Forcall(s) (8, 21, 22, 31), Gotall (21), Planalla (27), Tornallo $(25,27)$.

1.5. -án(s), -ano(s), -ana(s) (<-ānu, - $\bar{a} n a)$ : es un sufijo originariamente formador de adjetivos que indican pertenencia, pero en nuestros días lo encontramos en resultados totalmente lexicalizados (GIRALT 1995: 229):

1.5.1. aparece en sustantivados lexicalizados: Brainans, Capellans (14), Entuixano(s) (20, 25, 28), Esquilano, Estollans, Mediano (14, 17, 31), Miano (25), Solana(s) $(1,2,3,4,5,6,7,8,9,10,11,12,13,14,15,17,19,20,21,22,23,24,25,26$, 27, 28, 29, 31, 32), Solaneta(s) (gral.), Solano(s) (14, 20), Sobiranas (21)/ Subiranas (28).

1.5.2. Con un sentido despectivo lo encontramos en Artigorans (32), Cabellano (12), Cambrán (8), Camizán (18), Camparrano (27), Chuñán (16), Feixana(s) (9, 14, 
23), Faixano (1) / Feixano (3, 14, 23), Faixana (8), Feixans (9, 12), Fontanal (28), Forcanada (25), Mozán (8), Piñana (9), Quintán (20).

1.6. $-\operatorname{ar}(\mathbf{s})(<-\bar{a} r e)$ : de origen latino, pudo ser una variante de -āle $(>-a l)$ para formar adjetivos de pertenencia; hoy lo encontramos en sustantivos. Los valores anotados son los siguientes:

1.6.1. posee un valor locativo, especialmente para designar el lugar donde abunda una planta, arbusto o árbol: Aciar (3), Albar(s) $(25,32)$, Arbolar (6), Armudellar (26), Boixeguar (29), Caixigar (6, 9, 15, 19, 23), Canamar (9, 23), Canemar (9, 26, 28), Cañamar (12, 23), Ciscar (15) / Siscar (26), Coscollar (8, 12, 18, 29, 31) / Cuscullar (26), Cunillar (31), Chuncar (16, 27), Espigolar (15), Espinazar (18), el Llecinar (12, 27), Napar (10), Olivar (23), Parrar (8, 9) Pinar (1, 23, 25, 28), Queixigar (6, 9, 30), Rebollar (8), Rourar (13), Salencar (25), Salitar (23), Segalars (28).

1.6.2. Asimismo, forma sustantivos a los cuales da una idea de colectividad, además de un carácter locativo en algunos casos: Aguilar (16), Baradar (9), Basadá (22), Carpiná (22), Cillars (2), Comellar (28), Cunillar (31), Chordar (7), Panar (6), Llomar (30), Llosars (25), Molar (19), Molinar (2), Mollar(s) (6, 14), Nabar (9), Pallars (6, 18, 23), Rengar (26), Rinllar (12), Toscar (23), Villars (28), Visar (30).

1.7. -arda (<-hart): es un sufijo de origen germánico (ALVAR 1953, p. 269). Lo hemos registrado en Cañarda (23) y Codarda (18), con un valor aumentativo y despectivo, matiz que también se da en castellano y aragonés (GIRALT 1995, p. 230).

1.8. -arro, -arra $(<*$-arru): de origen prerromano, posiblemente vasco (ALVAR 1953, p. 264). Su sentido esencial es el despectivo, con un valor aumentativo como se observa en Prau Binarro (14), Fumarral (12), Cascarro (23), Cascarrals (derivado de Cascarro, 18), Cllotarrons (21), Picarrons (derivado de Picarro, 23), Pontarró (<Pontarro) (30), Puntarrón (<Puntarro) (15).

1.9. -ás, -aso(s) / -asso(s), -asa(s) / -assa(s), -az, -azo(s), -aza(s) (<-acěu): sufijo de gran productividad en castellano, catalán, gascón y aragonés (GIRALT 1995, p. 232). Originariamente, este sufijo formaba adjetivos denotando pertenencia o semblanza con alguna cosa. Otros valores que posee son los siguientes:

1.9.1. Carácter aumentativo: Cardigazas (12), Castellazos (2, 12, 23), Ferraz (9, 23, 27), Fornás (28), Paellaza (23), Planaza(s) (15, 19, 23), Rebollás (30), Terrasa (11).

1.9.2. A veces se une al sentido aumentativo cierto significado peyorativo: Aguilás (10), Argilassos (26), Arnazas (16), Artigasa (14), Cabanassa (29), Cadenasos (14), Campás (20, 21), Campaz (15), Campazo(s) (1, 9, 23), Canalazo (23), Cardigazas (12), Castellaso (14), Codaza (15), Comaza (18), Cornás (8), Coronaso (11), Costanás (8), Costanazas (9, 23), Costanazo(s) (7, 10, 15), Costeraza (12, 23), Costonazo (23), Covilassos (29), Cucurazas (23), Cugulaza (9), Chuntanazos (9), Estoscllasas (32), Fontanazas (4), Fornassos (5), Huertazos (12), Llanaza (9, 23), Llaunazo (18), Llanassas (29) / Llenassas (8), Llenasa (21), Llenaza(s) (9, 12, 23, 24, 27), Llenazos (23), Llinasas (26), Llinasos (28), Llitasos (25), Llusás (22), Olivassos (8), Padellasos 
Significado y valores de los sufijos en la toponimia ribagorzana aragonesa

(25), Paulassos (32), Penazos (3), Piñasa (22), Poblazos (10), Pocinazas (23), Ribasa (14), Rocasos (22), Royás (10), Serratiás (10), Solanassa (5), Solañás (22), Toñasas (22), Toscasos (6), Viñasa $(20,32)$ / Viñaza (12), Vinyasas (21), Vinyanyás (5).

1.10. -au < -ald: de origen germánico y valor eminentemente peyorativo (ROHLFS 1931, p. 140), está mínimamente atestiguado en nuestra toponimia, donde razones poderosas de orden analógico lo han reducido eliminando la consonante final (no hay más que pensar en la presencia abrumadora en tal posición del sufijo homófono -au<-atu que configura los participios de perfecto) (COROMINES 1991, p. 228). Sólo hemos anotado dos casos: Espinau (21), Moregau (22).

1.11. -dó(r), -dora, -dera(s): posee una doble procedencia latina:

1.11.1. <-tōre: designa el agente de la acción verbal, formando sustantivos que indican generalmente oficio: Munyidora (30), Teixidó (23), Triador (6).

1.11.2. <-tōriu, $-a$ : señala el lugar o instrumento donde se realiza la acción verbal: Abeurador (26) / Abrevador (18), Amurriadors (3, 21, 23), Cardadera (31), Esmoladera(s) (15, 23), Espaldador (23), Pinchadors (3). Baldeador (28), Cardadera (9), Filadera (20, 28), Llavaderas (28), Llavador (28), Mallador (6), Muidor(s) (14, 17), Pipidera (25), Posaderas (20), Regadera (6), Saladera (28), Saladero (28),

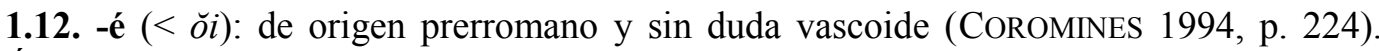
Únicamente hemos registrado dos ejemplos: Argoné (18), Bilsé (18).

1.13. -é, -et(s), -eta(s) (<-ĭttu): este sufijo se desarrolló en latín vulgar para constituir diminutivos de nombres propios de mujer y pronto adquirió gran fuerza expresiva llegando a ser uno de los más prolíficos en varias lenguas romances como el aragonés, catalán y provenzal. Según GONZÁLEZ OLLÉ, también debió ser el sufijo más frecuente en el aragonés medieval hasta el siglo XV, momento en que empezó a ser sustituido por -ico (GONZÁLEZ OlLÉ 1962, p. 145 \& 191). Actualmente, sigue siendo la formación autóctona en buena parte del dominio aragonés septentrional.

1.13.1. Su valor principal junto a sustantivos es el de diminutivo-afectivo: Aigueta (18), Algareta (16), Aubageta (26), Bancalet (12, 23), Baranetas (18), Barqueta (32), Barranquet (15), Barraqueta (3), Barrilet (29), Basalet (28), Baseta (9), Basetas (13), Basseta (21), Bodegueta(s) (23, 27), Bordeta (18), Brocadeta (15), Buigueta(s) (9, 12), Cabañeta (18), Campet (3, 9, 12, 22, 23), Canaleta(s) (3, 8, 9, 12, 13, 15, 18, 23), Carbaseta (22), Carransalet (6), Caseta(s) (3, 9, 12, 15, 18, 23), Castellet (19), el Clotet, els Clotets (12), Cogulet (26), Colladet (9, 18), Colladeta (12, 21, 23), Collassets (5), Comellet $(2,27,29)$, Cometa $(8,9,23)$, Comunet (23), Coroneta $(18,25)$, Corralet (12, 23), Corraleta (19), Covetas (3), Cotielleta (18) Cruceta (4, 16), Cuadranet (23), Cuadreta (23), Cuadronet (12, 24), Cuquet (3), Desenrunadet (23), Esplugueta (23), Esponalets (12), Ereta (3, 9, 23), Faixanetas (4), Faixetas (4, 9, 12, 23), Feixeta (23), Fontaneta (9, 23), Foradet (14), Forné (3), Fornets (19), Furquet (23), Gabarret (23), Gargalet (32), Garoneta (9), Gragüet (13), Lapayetas (3), Llanetas (9, 23), Llibonet (25), Lloseta (8), Medianet (12), Medianet (12, 23), Mollaret (12, 32), Montañeta (18), Montet 
(22, 31), Palanqueta (2), Pallaret (23), Palluceta (18), Paset (23), Pauletas (15), Peñetas (15), Planet (23), Pocineta (12), Purroyet (19), Puyadeta (12), Roqueta(s) (6), Siarreta (23), Sinsoletas (9), Sorteta (22), Tollet (31), Tozaleta (9, 23), Tuzaletas (7), Ventaneta (1), Vinyet (5).

1.14. -edo, -eda, -et, -eta (>-etum, -etam): posee un carácter colectivo, sobre todo en los casos que refieren fitónimos: Aliagaret (3), Artigueta (3, 18), Aspinalbeta (22), Barceta (23), Boixeda (13) / Boixeta (30), Caixagueret (23), Caixaguereta (9, 23), Calsedo (11), Cardoseta (23), Castañet (20), Cercedo (11), Corniereta (3), Curnyereta (2), Escobetas (3), Estelledo (11), Serra Fontsedo (28), Gabarret (9), Nugueret (12), Olivereta (23), Olsineta (30), Pinedo (11), Pineret (6, 28), Pomereta (12), Planedo (32), Solaseda (28).

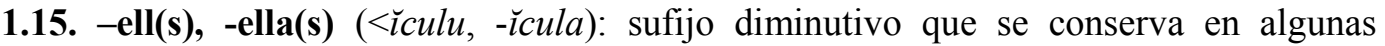
palabras tomadas del latín. Su carácter diminutivo pudo haberse perdido posteriormente en los topónimos registrados: Ansonella (29), Bardella (21), Basellas (26, 28), Bichella (15), Cabanella (30), Campella(s) (11), Cananella (24), Castisells (11), Comella (7), Comichellas (15), Congostell (30), Cordells (8), Cornella (24), Escuadrells (6), Foncinell (24), Fontanella(s) (20, 29, 31), Labunella (24), Llangostells (13), Peñella (23), Perelleta (9), Piñella (15), Planell (8), Pllanella (26), Portell (13, 29), Portella (13, 21), Pradella(s) (17, 31), Probellas (10), Regatell (2), Ribellas (28), Rocinell (13), Sarradells (7), Sirvella (24), Solanella (7), Tosquella (29), Vertella (25).

1.16. -enca: de origen germánico (MENÉNDEZ PIDAL 1989, p. 234) o prerromano (COROMINAS 1972, p. 187), parece tener un valor despectivo en Eixalencar (27), Ixalenca (28), Paulenca $(25,6)$, Salencar $(<$ Salenca) $(20)$.

1.17. -é, -er(s)/-és, -ero(s), -era(s) (<-arĭu, -ařa ): es de gran productividad en nuestra zona de estudio. Como indica Alvar, en su origen tenía un valor adjetivo, pero ya en latín sirvió para formar sustantivos (ALVAR 1953, p. 262-264). Los valores semánticos que posee son los siguientes:

1.17.1. forma nombres de árboles y de plantas: Acirés (9), Almendrera (19), Avellanés / Avellaners (26), Balaguera (7), Barcillera (30), Basanero (13), Bedero (9), Bitaguera (9), Boixiguer (8), Boixiguera (12, 23), Buixidero (27), Buixiguero (23), Canero (15), Cereceras (12), Cercadero (18), Coscolleras (24), Chunquera (16), Figuera (18), Garraberas (18), Manzanera (23), Morera (20), Noguero (23), Nuguero (9, 12), Oliveras (9, 23), Pallero (23), Pallés (27, 30), Perera(s) (10, 12, 28), Pomera (23), Pomero (25), Pruners (12), Rebollera (22, 30), Sarguera (24), Sarnera (18), Serbera(s) $(12,23)$, Teixonera (23), Vidigueras (8), Vinyers $(21)$.

1.17.2. Forma sustantivos que indican intensidad, lugar y situación y multitud del objeto nombrado por el primitivo: Agüeres (16), Aguileras (12), Baixadera (31), Balsero (27), Bayero (22), Cabanera (30), Cabrer (2), Canteras (3, 9), Carbonera (1, 21, 29), Cascarré (1, 15), Catera (23), Cibadero (23), Colomero (19, 24), Coroner (24), Chesera (16), Chesero (9, 19), Espaldadero (1), Estrimera (9), Estrimero (9), Falconera (32), Fanduguero (10), Ferrera (23), Fornera (32), Gravera (9), Lavanera (15), Lleners (12, 
Significado y valores de los sufijos en la toponimia ribagorzana aragonesa

21), Llenero (12, 18), Llenguadera (5, 32), Matera (19), Mosquera $(23,26,28)$, Osero (23), Palomero (23), Pedregeras (29), Peguera (2), Penero (13), Placeras (23), Pllanero (31), Ratés (22, 31), Reguer (30), Ringuera (7), Salineras (1, 18, 23, 30, 32), Salinereta(s) (3), Sarrallera (30), Senyaderos (5), Sulsiaders (8), Taberbera (14), Teulera (26), Terrero (16).

1.17.3. Indica relación con lo indicado por el primitivo: Arnero (12, 23), Barreras (3), Cabañera (12, 23), Calera (18), Carcadera (9), Carrera (22), Codera(s) (9, 23), Codero(s) (23), Costera (12, 18, 23), Chugadero (9, 12), Esmoladera (23, 24), Farné (13), Fiteros (23), Gallinero (18), Llavanera(s) (9), Llongadera(s) (9, 18, 23), Llosero (25), Morrera (3, 9), Palomera (24), Palomeres (25), Pandera (9), Planeras (23), Planero (12), Porquero (25), Rallera (23), Rallero (12), Regalera (12, 20), Rialera (23), Ribera (9, 23), Serenero (23), Solanera (3), Solanero (23), Terrero(s) (12, 23), Tornacé (13), Vidaller (23), Villero (24), Viveros (23).

1.18. -ería(s) $(<-a r \breve{u}+-i a)$. Su valor principal es el de indicar el lugar donde se realiza una actividad concreta: Farrerías (8), Ferrería(s) (9, 18), Tellería (4), Tenallería (4), Tejería $(18,23)$, Teulería $(7 / 30)$.

1.19. -és $(<-\bar{e} n s e)$. Sufijo utilizado para formar sustantivos de procedencia o residencia: Barrabés (23), Corzanés (23).

1.20. -iello, -ialla(s), -iella(s), -iaso, -iesa(s) $(<-e ̌ l l u, ~-e ̌ l l a ~)^{7}$. Presenta un comportamiento semejante al castellano y su carácter puramente de diminutivo se manifiesta en Angunielles (25), Aviturialla (9), Basarialla (18), Batasialla (25), Bunsiello (28), Cabanielles (25), Campiello (19), Casiallas (27), Casiasas (22), Castiello(s) (10, 25), Codadialla (23), Combiello (3), Comialla(s) (9, 23), Comialla(s) (6, 10, 15, 24), Comiella (15), Comiello (3, 4, 12, 27), Corniella (24), Cotiella (3, 15), Crusialla (28), Cubiello (6), Escaldialla (27), Espaniellas (6), Espardiallas (1), Espusiallas (6), Estiviella (18), Fonciellas (4), Fontisialla (11), Fontisiellas (11), Fontsiello (20), Funsiellas (20), Gradiello (15, 19), Laguniallas (25), Murillo de Liena (23), Moliniello (12), Montisiello (6, 28), Paciella (14), Pardiallas (23), Paretiallas (9), Perialla (27), Piniello (12), Pociello (12, 13, 29), Pontisiello (25), Portialla (9), Comapradiello (12), Puyatiello (23), Puyilella (20), Rodiella (25), Rubialla (23), Sarradiello (23, 28), Sarravadialla (12), Sorbaniella (19), Tarradiello (28), Tormiello (9, 12), Torrociella (15), Tosquialla (23) / Tosquiella (17), Pasaturbiello (31), Vadiello (25).

1.21. -ico $(<-\bar{\imath} c c u)$ : es considerado como el sufijo característico del castellano de Aragón para formar diminutivos, a pesar de que sólo a partir de mediados del siglo XV supera en frecuencia al morfema -et. Actualmente, es el sufijo más difundido por las provincias de Zaragoza y Teruel, llegando incluso a la provincia de Huesca, a pesar de que en una proporción muy baja. En la Ribagorza, como prácticamente en toda la provincia de

\footnotetext{
${ }^{7}$ Sobre la evolución de $\breve{\mathrm{E}}$ a ia, ie y -LL- a $-s$ - puede consultarse el trabajo de ALVAR (1953, p. 146-148) VÁZQUEZ OBRADOR (1992, p. 1062, 1112-1122).
} 
Huesca, se utiliza más - et. Los casos registrados, en los que apreciamos un valor afectivo, son pocos y se refieren algunos a antropónimos: Añica (23), Arnaldico (17), Pericó (21), Molica (14), Ramonico (21), Tomico (18), Toñico (30).

1.22. -iga (<-īca): de probable origen celta, persiste en topónimos como Artiga (s) $(1,2$, $3,4,5,6,7,9,11,13,15,17,18,19,21,22,25,27,28,29,30,31,32)$ у Buiga(s) $(<$ Bodiga(s) $(<*$ Boudīca) $(9,12,22)$ si bien encontramos un Bodidas $(19)$.

1.23. -il $(<-\bar{l} l e)$ : sugiere referencias locativas y, por extensión de éstas, de lugar donde se guarda algo: Ancils (25), Bañils (27), Cabanils (12), Cortils (12, 15, 18, 27), Leril (14), Pletil (11) / Plletil(s) (9, 12, 14, 18), Sancils (18), Tamarils (5).

1.24. -ils, -ill(s), -illo, -illa (<-īculu, - īcula): sufijo diminutivo, que en algunos casos conserva la $-o$ final (TERRADO 2008, p. 69), y que ya poseía este valor en latín. Este carácter diminutivo se ha ido manteniendo, si bien observamos también un matiz despectivo en algunos de los topónimos anotados: Barranquill $(21,26)$, Cabecillo (18), Caixigarillo (23), Campillón(s) (<Campillo(s)) (11, 15), Campurrillo (21), Cananillo (4), Canarils (29), Canarill (8) / Canarillo (22), Canarills (26), Carrodilla (16), Casilla (3), Castesillo (9, 13), Cepillo (<cepa) (24), Cerillo (30), Clavillas (22), Congostillo (29), Cortills (12), Cubills (31), Cusillo (14), Escalmadillo (27), Fontanilla (24), Pinarillo (3, 21), Pontillo (9,11), Portillo (24), Pllanilla (28), Pradillo (20), Salterillo (25), Torrecilla (16), Tosquilla (21), Villilla (23).

1.25. -ín(s), -ino(s), -ina(s) $(<-\bar{i} n u, \quad i n a)$ : originariamente tenía el sentido de «perteneciente a », valor que conserva en: Abadina (18), Ballumins (5), Calcinas (2) / Calsina (6), Collabardina (3), Carcín (23), Cervín (3, 9), Corrongolina (12), Costa Llobina (28), Forcalins (20), Frechinas (16), Llerín (24), Padarnín (12), Pardina (11), Pellina (25), Planderins (23), Pocino(s) (18, 23) / Posino (28), Posina (28), Posolobino (25), Pradina(s) (3, 6), Raixín (3), Salina (28), Sentins (5).

1.26. -iso(s) -isa(s), -izo, -iza(s) (<-īcium, ìciam): de escasa productividad en la toponimia registrada, originariamente tenía un valor adjetival, y expresa pertenencia, participación o propiedad en un objeto (GIRALT 1995, p. 242). También se suele intuir un cierto valor peyorativo: Callarizas (19), Callerisa (14), Callizo (16, 23), Pallisa (14), Pedrisa(s) (11, 28), Rompedisas (17), Tremolisos (28), Vaquirisa (11), Vaquerisal $(<$ Vaquerisa) (14).

1.27. -ís, -iu (<itze): de origen prerromano, seguramente vasco, tiene un valor colectivo. Los ejemplos que hemos anotado son dos: Carriu (28), Cheris (28).

1.28. -ko: ampliamente documentado en vasco, indica 'posesión' o "pertenencia » (AZKUE 1969). Así se manifiesta en los topónimos Burrinyaco (26), Gabesaco (23), Rubiñaco (27), Vinyaco (8), 
Significado y valores de los sufijos en la toponimia ribagorzana aragonesa

1.29 -ó, -ón(s), -ona(s) (<-ōne): su significación original debió ser la de una individualización neutra. Desde aquí, llegó a indicar una variedad individual de una persona $\mathrm{u}$ objeto: en catalán, francés y provenzal tomó rápidamente un carácter diminutivo; en español e italiano desarrolló un valor aumentativo, mientras que en los dialectos pirenaicos se produjo una extraña confusión de conceptos y se dieron ambas posibilidades (GIRALT 1995, p. 242-243). En los ejemplos que hemos documentado, posee dos valores claros:

1.29.1. normalmente posee un valor aumentativo-despectivo: Abarcón (15), Albezons (23), Angón (18), Arañó (22), Arañona (12), Armellons (19), Aviñón (28), Barrillón (3), Basó (8, 28), / Basón(s) (9, 12, 15, 23), Biñón (13), Bobón (10), Bocona de Dalt (17), Buigón (9, 12, 22, 23), Campazón (9, 23), Campillon(s) (9, 12, 14, 23), Canalón (3, 23), Cantón (3, 19), Carrascalón (23), Castillón (18, 23), Cibadalons (12), Cllavillons (31), Clusón (3), Corralón (9, 23), Corllatons (25), Coscollón (18), Costerons (13), Cotón (24), Cuadrón (9, 12, 13, 27), Chinebralón (23), Escllusons (19), Escrions (28), Esplugons (12), Fogueró (8), Fondón (27), Fornón(s) (6, 9, 31), Fovón (19), Frontón (15), Hortón(s) (27, 28), Huertons (24), Labayons (23), Lapayons (3), Llanetons (23), Llecinarona (24), Mallona (3), Macetón (24), Mollarón (22), Molló (29) / Mollón (13), Morrón(s) (1, 12), Olivarons (23), Palancón (23), Paletons (14), Pallerón (10), Perillón (20), Comaperona (9), Picarrons (23), Pilonas (3), Pinarón (9, 12), Pisons (12), Portillon (25), Pugañons (12), Puntarrón (4, 14), Puntons (9), Puyadón (23), Quixalón (9), Rallerón (12), Redolons (1), Rosón (3), Serrón (14), Tozalón (23) Tuzalón (3), Vallón $(18,23,25)$ Vedadons (23), Vergarón (12), Villarón (31).

1.29.2. En otros casos no podemos descartar el valor diminutivo: Arneró (26), Arnerons (15), Artigón(s) (3, 11, 15, 23, 29), Bancalón(s) (26, 32), Comellón(s) $(26,32)$, Olivó (8, 19), Picó $(16,29)$ / Picón (9, 23), Pletillón (3) Pradillón(s) (12), Vallón $(18,23)$.

1.30. -oga/-uga ${ }^{8}$ : posee un sentido de colectividad en Abellanuga (22), Fornoga (22), Pedroga (22), Tremologa (32).

1.31. -ol(s), -ola(s), -uelo, -uela, -uala(s) (<-ŏlu, ŏla): en latín poseía un valor diminutivo (GIRALT 1995, p. 244) todavía apreciable en algunos topónimos lexicalizados recogidos: Arguala (13), Bagüeñola (14), Bardiguala (28), Bordualas (22), Cabazola (13), Cabeçolas (29, 30), Caixolas (30), Canerol (26), Carrigüala (9), Carrerol (27), Ciaruelo (23), Ciercol (15), Ciñualas (24), Cirisueles (25), Cornuchuelo (3), Cunyols (29), Cruzillada (14), Chabarruelo (16), Eglesiola (24), Escarriguala (10), Espígols (30), Faixolas (24), Faixuelo (23), Feixualas (18), Figuerol (29), Fuixañolas (27), Maiguala(s) (6, 13, 28), Maigüelo (31), Mallols (24), Malluelo (12), Miargüles (14), Moscuruela (25), Muixola (13), Muzuelo (15), Pallarol (4), Pallaruelo (10, 16), Pigüelo (14, 31), Pozuelo (27), Poyuelo (19), Puértolas (16), Puyuelo (23, 11, 28), Regachol (19), Rigüelo (18), Prigüala (9), Tarrazuala (23), Tussola (29), Ventallola (25).

\footnotetext{
${ }^{8}$-oga y - uga responden a una evolución particular del sufijo colectivo - osa que perdería la $-s$ - intervocálica y posteriormente se produciría un fenómeno de epéntesis de una - $g$ - intervocálica (VÁZQUEZ 2005, p. 13).
} 
1.32. -ós, -osa(s), -oses, -oso, -uso, -ús $(<-\bar{s} u)$ : ofrece una idea de abundancia en un grado supremo, si bien en algunas ocasiones puede aportar también un claro sentido despectivo: Abetosa (11, 25), Albarosa (12, 25), Pialbaruso (18), Aliagosa (27), Ardillosa (27), Arguilosa (32), Arsosa (6), Bardosa (31, 32), Cadenosas (27), Caixigosa (13), Cardosas (28), Castricosas (31), Cercosa (28, 31), Cercús (18), Comartosa (31), Comarzosa (23), Comatosa (15), Corroscuso(28), Coscollosa (27), Cuellobrotuso (23), Escarpinosa (25), Espigantosa (14), Espigolosa (18), Espinantosa (14), Felegrosa (11, 17, 20), Fenellosas (21), Fornigosa (21), Fornosa (23), Gabardosa (31), Gabarroa (<Gabarrosa) (21), Gabarrosa (1) / Gavarrosa (24, 27), Gargallosa (25), Gargalluso (18), Garrabosa (18), Llanosa (26), Llanticosa (16), Marañosa (28) / Mariñosa (9, 12, 23), Mascarosa (30), Matosa(s) (11, 16, 21, 27), Moixoses (7), Palentosa (6), Palleroa (<Pallerosa) (21), Pedregosa (4), Pedrosa(s) (13, 21, 30), Pinosa (18, 23), Queixigosa (22), Quixigós (7), Rebollosa (30), Sabartosa (14), Santigosa (23), Savinós (29), Segalosas (28), Tormegosa (25), Tortellosa (26), Trabanoses (5), Tremolosa (11), Tremosa (5), Turmosa (11), Ventosa (3).

1.33. -ot, -ota (<-ŏttu): primitivamente tenía una función de diminutivo, pero su antiguo valor ha desaparecido (GIRALT 1995, p. 245). Lo encontramos con un marcado valor aumentativo-despectivo en Badinota (15), Basota (31), Boigot (2), Camparrot (32), Campot (22), Canota (13), Carantot (29), Casalot (29), Casota (26), Castellot (8), Erota (12), Montot (32), Pallerot (23), Pasterot (8), Pinarot (12), Pradot (31), Renguerot (7), Sarradot (21), Serradot (5), Terrota (5), Torrota (26), Tussalot (32), Viñota (23).

1.34. -ué $(<\breve{o i})$ : de origen prerromano, seguramente vascónico. Aparece en varios topónimos mayores (MARTín DE LAS PUEBLAS 2003, p. 472) (Ardoné (25), Chisnegüe (20), Erisué (14), Negüé (14), Pinarrué (11), Ramastué (6), Renanué (6), Sargüé (6), Sesué (20)) y en topónimos menores (Llisué $(15,27)$.

1.35. -ullo, ulla (<-uculu, -ucula): parece tener un valor diminutivo en Cogullo (28) y Cogulla (28).

1.36. -ún $(<*$-uin $<*$ oin): probablemente de origen ibero-vasco (MichelenA 1961, p. 507), su valor semántico es el de « al pie de »: Esterún (9), Larún (23), Laspún (23), Naspún (23). En este sentido, hay una gran cantidad de lugares de la provincia oscense en el que la presencia del sufijo -un aparece en ls formsción de macrotopónimos: Astún, Asún, Astún, Cercún, Embún, Estaún, Gordún, Laruns, Navardún, Presún, Rapún, Sahunc, Secorún, Sercún, Sescún, Sesún, Sisún, por ejemplo.

1.37. -uz (<-ucĭus): según COROMINES (1994, p. 413) se trata de un sufijo que suele aparecer, en primer lugar, junto a sustantivos y adjetivos de la lengua común, y en segundo lugar, junto a antropónimos como es el caso de Garuz (23) que procedería de una base KARUCIUS o CHERUCIUS (COROMINES 1994, p. 413). 
1.38. -ús, -uzo, -uza $(<\bar{u} c e u)$ : de evidente valor peyorativo tal como observamos en Palluzeta (<Palluza) (18), Pocinuzo (23), Terrús (20).

\section{Conclusiones.}

Esbozado el panorama de sufijos que presenta la toponimia ribagorzana, podemos realizar las siguientes conclusiones:

2.1. El sufijo diminutivo con mayor vitalidad en nuestra toponimia es -et(s), - eta(s), -é seguido, pero con un número menor de frecuencias, de -ell(s), -ella(s); -ils, ill(s), -illo, -illa; -ialla(s), -iella(s), -iello y sus variantes -ieso, -iasa(s); -ó, -ón(s), -ona(s) y-ol(s), -ola(s), -uelo, -uela, -uala(s). El significado inicial de tales sufijos es, sin duda, el de aminorar el significado del primitivo al cual se juntan, pero qué duda cabe que no podemos olvidar los valores subjetivos-emocionales que en éstos aparecen.

2.2. El cuadro de los sufijos puramente aumentativos es menor que el de los diminutivos. Podemos destacar los sufijos acho, -acha(s); -arda; -arra, -arro; -ón(s), -ona y, sobre todo, -ás, -aso(s) / -asso(s), -asa(s) / -assa(s), -az, -azo(s), -aza(s) y -ot, - ota. En todos ellos, pero especialmente en los dos últimos, es difícil diferenciar ese valor aumentativo de una clara noción de despectividad.

2.3. El formante despectivo-peyorativo es fundamental, como ya hemos dicho, en número considerable de sufijos que aparecen en la toponimia ribagorzana. Nos estamos refiriendo, sobre todo, a sufijos como -ás, -aso / -asso(s), -asa / -assa, -az, -azo(s), -aza(s); -ó, -on(s), -ona y -ós, -osa(s), -oses, -oso, -uso, -ús y, con un número menor de frecuencias respecto a éstos, -án(s), -ano, -ana; -arda; -arra, -arro; -enca; -ils, -ill(s), -illo, -illa; -ot, -ota y -us, -uzo, -uza.

2.4. Los sufijos $-\dot{e}$, -er(s)/-és, -ero(s), -era(s) y -ar(s), que aparecen en un buen grupo de topónimos, son básicamente locativos al indicar nociones, por ejemplo, como el lugar donde hay abundancia de determinadas plantas y árboles.

2.5. Por último, también el caso de la sufijación se puede observar el carácter puramente mixto de las hablas ribagorzanas. Así observamos elementos comunes al altoaragonés y al catalán como el sufijo - et(s), -eta(s). Rasgos puramente aragoneses son los aumentativos-despectivos -ón(s), -ona y, -aso / -asso(s), -asa / -assa, -az, -azo(s), - aza(s). Y a la influencia catalana se deben los sufijos - ell(s), -ella(s) y ot, -ota y el aumentativo -ás. Todo ello hace que hablemos de una zona lingüística de enorme importancia para el estudio de la toponimia románica por cuanto podemos observar en los nombres de lugar, verdaderos fósiles de la evolución de las lenguas, rasgos propios de las hablas que confluyeron y confluyen en este amplio espacio geográfico que es la Ribagorza. 


\section{Bibliografía.}

\section{Colección Toponimia de Ribagorza.}

1. Porras, Encarna \& Terrado, Javier. 2001. Toponimia de Ribagorza. Municipio de Santaliestra y San Quílez. Lleida: Universitat de Lleida.

2. TERRAdO, Javier. 2001. Toponimia de Ribagorza. Municipio de Arén. Lleida: Universitat de Lleida.

3. Selfa, Moisés. 2001. Toponimia de Ribagorza. Municipio de Campo. Lleida: Universitat de Lleida.

4. Rizos, Carlos Ángel. 2001. Toponimia de Ribagorza. Municipio de La Puebla de Castro. Lleida: Universitat de Lleida.

5. Bordas Pallás, A. 2002. Toponimia de Ribagorza. Toponimia de Ribagorza. Municipio de Montanuy. Lleida: Ed. Milenio.

6. Martín De Las Pueblas, Jesús \& Hidalgo, María Asunción. 2002. Toponimia de Ribagorza. Municipio de Castejón de Sos. Lleida: Ed. Milenio.

7. VÁzQUEZ, Jesús. 2002. Toponimia de Ribagorza. Municipio de Lascuarre. Lleida: Ed. Milenio.

8. Francino, Gloria. 2002. Toponimia de Ribagorza. Municipio de Sopeira. Lleida: Ed. Milenio.

9. SelfA, Moisés. 2002. Toponimia de Ribagorza. Municipio del Valle de Bardaixín. Lleida: Ed. Milenio.

10. Rizos, Carlos Ángel. 2002. Toponimia de Ribagorza. Municipio de Secastilla. Lleida: Ed. Milenio.

11. Martín De Las Pueblas, Jesús \& Hidalgo, María Asunción. 2002. Toponimia de Ribagorza. Municipio de Chía. Lleida: Ed. Milenio.

12. Selfa. Moisés. 2002. Toponimia de Ribagorza. Municipio de Valle de Lierp. Lleida: Ed. Milenio.

13. VÁZQUEZ, Jesús. 2002. Toponimia de Ribagorza. Municipio de Capella. Lleida: Ed. Milenio.

14. Martín De Las Pueblas, Jesús \& Hidalgo, María Asunción. 2002. Toponimia de Ribagorza. Municipio de Sahún. Lleida: Ed. Milenio.

15. Porras, Encarna, Terrado, Javier \& VÁzQUeZ, Jesús. 2002. Toponimia de Ribagorza. Municipio de Perarrúa. Lleida: Ed. Milenio.

16. VÁZQUEZ, Jesús. 2002. Toponimia de Ribagorza. Municipio de Estadilla. Lleida: Ed. Milenio.

17. Martín De Las Pueblas, Jesús \& Hidalgo, María Asunción. 2002. Toponimia de Ribagorza. Municipio de Villanova. Lleida: Ed. Milenio.

18. Selfa, Moisés. 2004. Toponimia de Ribagorza. Municipio de Seira. Lleida: Ed. Milenio.

19. Rizos, Carlos Ángel. 2004. Toponimia de Ribagorza. Municipio de Graus. Zona de Jusseu, Aguilaniu y Torres del Obispo. Lleida: Ed. Milenio.

20. Martín De Las Pueblas, Jesús \& Hidalgo, María Asunción. 2004. Toponimia de Ribagorza. Municipio de Sesué, Lleida: Ed. Milenio.

21. Terrado, Javier \& RourerA, María Cristina. 2005. Toponimia de Ribagorza. Municipio de Monesma y Cajigar (Monesma i Quixigar). Lleida: Ed. Milenio.

22. VÁZQUEZ, Jesús. 2005. Municipio de Veracruz, Lleida: Toponimia de Ribagorza. Ed. Milenio.

23. Selfa. Moisés. 2005. Municipio de Foradada de Toscar. Lleida, Ed. Milenio.

24. Rizos, Carlos Ángel. 2006. Toponimia de Graus, II. Zonas de Barasona, Graus y Panillo, Lleida: Toponimia de Ribagorza. Ed. Milenio.

25. Martín De Las Pueblas, Jesús \& Hidalgo, María Asunción. 2006. Municipio de Benasque. Lleida, Ed. Milenio. 


\section{Significado y valores de los sufijos en la toponimia ribagorzana aragonesa}

26. Terrado, Xavier. 2007. Toponimia de Ribagorza. Toponimia de Puente de Montañana. Lleida: Ed. Milenio.

27. Rizos, Carlos Ángel. 2007. Toponimia de Ribagorza. Toponimia de Graus, III. Zona de Fantova. Lleida: Ed. Milenio.

28. Martín De Las Pueblas, Jesús \& Hidalgo, María Asunción. 2008. Toponimia de Ribagorza. Municipio de Bisaúrri. Lleida: Ed. Milenio.

29. Terrado, Javier. 2008. Toponimia de Ribagorza. Toponimia de Ribagorza. Municipio de Viacamp y Literá. Lleida: Ed. Milenio.

30. Rizos, Carlos Ángel. \& Selfa. Moisés. 2009. Toponimia de Ribagorza. Municipio de Benavarri. Lleida: ed. Milenio.

31. VÁzQUEZ, Jesús. 2010. Toponimia de Ribagorza. Municipio de Torre la Ribera. Lleida: Ed. Milenio.

32. Francino, Gloria \& FeIXA, Carmen. 2011. Toponimia de Ribagorza. Municipio de Bonansa. Lleida: Ed. Milenio.

\section{Otros títulos citados a lo largo de este trabajo}

Alonso, Amado. 1967. « Noción. Emoción. Acción y Fantasía en los diminutivos ». Estudios Lingüisticos. Temas Españoles. Madrid: Gredos, p. 161-189.

ANDOLZ, Rafael. 1992. Diccionario aragonés. Zaragoza: Mira Editores.

Alvar, Manuel ,1949. « Toponimia del alto valle del río Aragón ». Pirineos 5, p. 389-496.

- 1953. El dialecto aragonés. Madrid: Gredos.

ARNAL, María Luísa. 1986. "Notas sobre la sufijación apreciativa en La Puebla de Castro (Huesca) ». Archivo de Filología Aragonesa 38, p. 67-88.

AzKue, M. R. 1969. Diccionario Vasco-Español-Francés. Bilbao: Editorial La Gran Enciclopedia Vasca.

BAdia MARgarit, Antonio. 1949. « Mal, roca en la toponimia pirenaica ». Actas de la Primera Reunión de Toponimia Pirenaica. Zaragoza: CSIC, p. 163-190.

BALlarín, Ángel. 1979. Diccionario del benasqués. Zaragoza.

BuESA, Tomás. 1989. "Sufijación apreciativa en ayerbense ». Estudios Filológicos aragoneses. Zaragoza: Universidad de Zaragoza, p. 113-134.

Corominas, Joan. 1972. Tópica Hespérica. Segunda edición. Madrid: Gredos.

COROMINES, Joan. 1991. El parlar de la Vall d'Aran (Gramática, diccionari i estudis lexicals sobre el gascó). Barcelona: Curial Edicions. 1994. Onomasticon Cataloniae 2. Barcelona: Curial Edicions.

ElCOCK, W. D. 1949. « Toponimia menor en el Alto Aragón ». Actas de la Primera Reunión de Toponimia Pirenaica. Zaragoza: CSIC, p. 77-118.

Francino, Gloria. 2005. Toponimia de Ribagorza. Municipio de El Pont de Suert. Lleida: Pagès Editors.

GARCíA BLANCO, Manuel. 1949. « Contribución a la toponimia aragonesa medieval ». Actas de la Primera Reunión de Toponimia Pirenaica. Zaragoza: CSIC, p. 119-143.

GIRALT, Javier. 1995. «Prexixos i sufixos a la parla de Sant Estece de Llitera (Osca) ». Archivo de Filología Aragonesa 51, p. 217-252.

GONZÁlez OlLÉ, Fernando. 1962. Los sufijos diminutivos en castellano medieval. In : Revista de Filología Española anejo 75. Madrid: CSIC.

KuHn, A. 1978. « Der Hocharagonesische Dialekt ». Revue de Linguistique Romane 1, p. 1-312.

Martín DE LAS Pueblas, Jesús. 2002. Toponimia del valle de Benasque. Universitat de Lleida: Tesis doctoral. 


\section{Nouvelle Revue d'Onomastique $n^{\circ} 53$ - 2011}

MenÉndez PidAl, Manuel. 1989. Manual de Gramática Histórica Española. Madrid: EspasaCalpe.

MichelenA, Luis. 1961, Fonética histórica vasca. San Sebastián: Diputación Provincial de Guipúzcoa.

Monge, Fernando. 1965. «Los diminutivos en español ». Actes du Xe Congrès International de Linguistique et de Philologie Romanes (Strasbourg 1962). París: Klincksiek, p. 71-78.

MotT, Brian. L. 1984. Diccionario Chistavino-Castellano. Zaragoza: Caja de Ahorro de la Inmaculada.

Rizos, Carlos Ángel. 2001. Toponimia de la baja Ribagorza occidental. Universitat de Lleida: Tesis doctoral.

ROHLFS, Gerhard. 1931. «Beiträge zur Kenntnis der Pyrenäenmundarten ». Revue de Linguistique Romane 7, p. 119-169.

— 1985. Diccionario dialectal del pirineo aragonés. Zaragoza: Instituto Fernando El Católico. . 1988. «Los sufijos en los dialectos pirenaicos ». Archivo de Filología Aragonesa 40, p. $115-170$.

SAurA, José Antonio. 2008. Toponimia de Aso, Yosa y Betés. Huesca: Universidad de Zaragoza, Prensas Universitarias, Xordica.

SElfa. Moisés. 2002. Toponimia del Valle Medio del Ésera (Huesca). Estudio lingüístico. Lleida: Pagès Editors.

TERRADO, Javier. 1992. Toponimia de Betesa. Lleida: Institut d'Estudis Il·lerdencs.

- 1998. «El elemento -navar(r) en la toponimia pirenaica y el nombre de Navarra ». Toponimia. Más allá de las fronteras lingüísticas. Lleida: Universitat de Lleida, p. 53-67.

VÁzQUEZ, Jesús. 1992. Toponimia de la Tierra de Biescas y Sobrepuerto. Huesca: Instituto de Estudios Altoaragoneses.

- 2008. Los nombres y la tierra: onomástica de Eriste, Sahún y Eresué. Huesca: Universidad de Zaragoza, Prensas Universitarias, Xordica. 